\title{
Development and Implementation of a Rotating Nanoimprint Lithography Tool for Orthogonal Imprinting on Edges of Curved Surfaces
}

\author{
Shraddha Supreeti ${ }^{1}\left[\right.$ ? Ralf Schienbein ${ }^{2} \cdot$ Patrick Feßer $^{3} \cdot$ Florian Fern $^{4} \cdot$ Martin Hoffmann $^{5} \cdot$ Stefan Sinzinger $^{3}$
}

Received: 18 March 2021 / Revised: 27 July 2021 / Accepted: 1 August 2021 / Published online: 18 August 2021

(c) The Author(s) 2021

\begin{abstract}
Uniform molding and demolding of structures on highly curved surfaces through conformal contact is a crucial yet oftenoverlooked aspect of nanoimprint lithography (NIL). This study describes the development of a NIL tool and its integration into a nanopositioning and nanomeasuring machine to achieve high-precision orthogonal molding and demolding for soft ultraviolet-assisted NIL (soft UV-NIL). The process was implemented primarily on the edges of highly curved plano-convex substrates to demonstrate structure uniformity on the edges. High-resolution nanostructures of sub-200-nm lateral dimension and microstructures in the range of tens of microns were imprinted. However, the nanostructures on the edges of the large, curved substrates were difficult to characterize precisely. Therefore, microstructures were used to measure the structure fidelity and were characterized using profilometry, white light interferometry, and confocal laser scanning microscopy. Regardless of the restricted imaging capabilities at high inclinations for high-resolution nanostructures, the scanning electron microscope (SEM) imaging of the structures on top of the lens substrate and at an inclination of $45^{\circ}$ was performed. The micro and nanostructures were successfully imprinted on the edges of the plano-convex lens at angles of $45^{\circ}, 60^{\circ}$, and $90^{\circ}$ from the center of rotation of the rotating NIL tool. The method enables precise imprinting at high inclinations, thereby presenting a different approach to soft UV-NIL on curved surfaces.
\end{abstract}

Keywords Nanoimprint lithography $\cdot$ Rotating NIL tool $\cdot$ Curved surface $\cdot$ Imprinting $\cdot$ Nanopositioning

\section{Introduction}

The patterning on curved or non-flat surfaces has gained prominence in recent years because of its rapidly increasing applications in the fields of optics, photonics, biomedicine, flexible electronics, solar cells, and others[1,2]. Over the years, different techniques, such as the use of gasbag

Shraddha Supreeti

shraddha.supreeti@tu-ilmenau.de

1 Electronics Technology Group, Technical University of Ilmenau, Ilmenau, Germany

2 Institute for Design and Precision Engineering, Technical University of Ilmenau, Ilmenau, Germany

3 Technical Optics Group, Technical University of Ilmenau, Ilmenau, Germany

4 JUMO GmbH \& Co. KG, Fulda, Germany

5 Microsystems Technology Group, Ruhr-University Bochum, Bochum, Germany pressure chambers and preshaping films [3, 4], have been adopted for imprinting on non-planar surfaces.

However, the stamps in these processes are bent to conform to the curvature of substrates. Therefore, the molding and demolding are not orthogonal to the surface. One of the major factors responsible for a successful nanoimprint lithography (NIL) process is demolding [5, 6]. Demolding is a crucial step during the solid phase or the post-ultraviolet (UV) curing phase of the NIL process. A problem arises when shear stress is induced during the separation of stamps and resist-coated substrates. Such stress can lead to structure distortion and pattern collapse, particularly in cases involving structures with high aspect ratios. For high-resolution structures with a sub-100-nm dimension, the high internal stress generated in their small cavities can weaken the rigidity of polymers and lead to deformation. Internal stress is therefore an inevitable part of the demolding process. Thus far, a few methods have been implemented to reduce stress by reducing surface adhesion; such methods include coating stamps with anti-sticking layers or using alternative stamp 
materials with low surface energy that makes the stamp surface hydrophobic [5].

In the age of interdisciplinary research, the integration of multidisciplinary fields allows the exploration of and experimentation on different approaches to addressing challenges. The simplicity of the soft UV-NIL process makes it suitable to be integrated into a nanopositioning and nanomeasuring machine (NMM-1). The NMM-1 is a coordinate measuring machine that has a positioning resolution of less than $0.1 \mathrm{~nm}$ and a working range of $25 \mathrm{~mm} \times 25 \mathrm{~mm} \times 5 \mathrm{~mm}$, [7-9]. To achieve nanometer accuracy, the machine utilizes the threedimensional Abbe comparator principle. On the basis of this principle, the machine performs position measurement with three laser interferometers that are virtually crossing one point of a thermally and geometrically stable mirror corner made of Zerodur (Fig. 1a). The accurate positioning capability of the NMM-1 tool allows the precise control of the effect of stamp deformation during the imprinting procedure.

Unlike conventional lithography tools, soft UV-NIL is preferable because it is not dependent on complex machines, strict environmental conditions, or optical limitations. In the current work, a compact and adjustable soft UV-NIL process was initially designed, assembled, and integrated into the NMM-1 [10]. The successful implementation of the fundamental process in the NMM- 1 created a foundation for making an NIL tool to be combined with a rotating device and integrated into the NMM-1. The combination enables five degrees of freedom of motion for orthogonal molding and demolding on edges of curved substrates.

\section{Device and Process Development}

\subsection{Rotating Nanoimprint Tool}

The positioning of a substrate is based on three independent linear movements in a Cartesian coordinate system of the NMM-1. Moreover, a fixed nanoimprint tool limits the addressable substrate geometries. An enhancement is introduced through the implementation of the ultraprecise rotational movements of the NMM-1 tool while keeping the precision within the nanometer range. Kinematics with a high degree of fulfillment consider a common instantaneous center of rotation in the tool center point (TCP). The integrated kinematic is based on the serial combination of an L-611 precision rotation stage (2018: L-611.9ASD, PhysikInstrumente GmbH \& Co. KG) and a WT-90 motorized precision goniometer (2018:WT-90(65509201), PhysikInstrumente GmbH \& Co. KG)(Fig. 1a).

The additional tool rotations of $360^{\circ}$ around $\Phi_{z}$ and $90^{\circ}$ around $\Phi_{x, y}$ of the NMM- 1 enable the hemisphere to be positioned orthogonally to its surface. As the TCP corresponds to the intersection of the three interferometers (a)

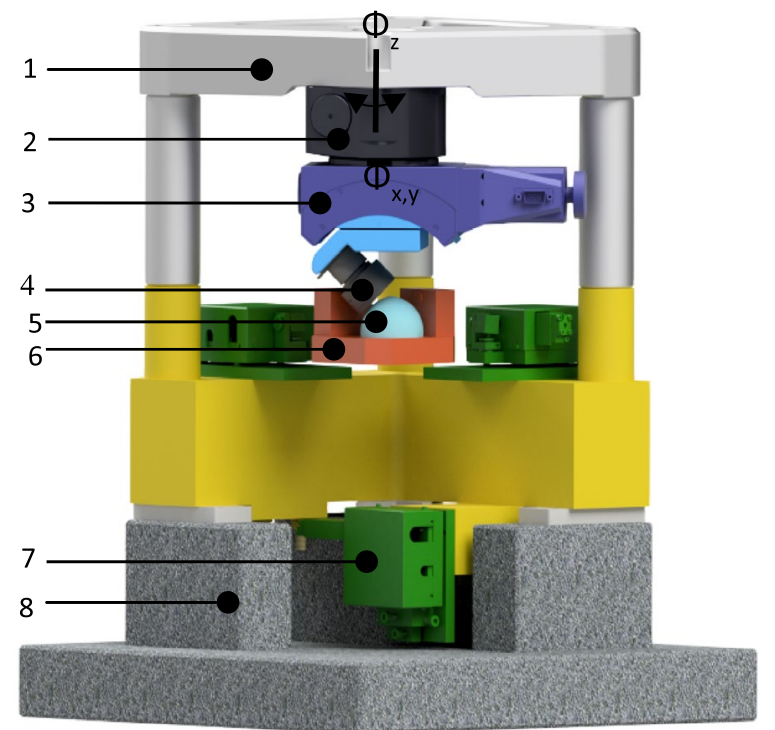

(b)

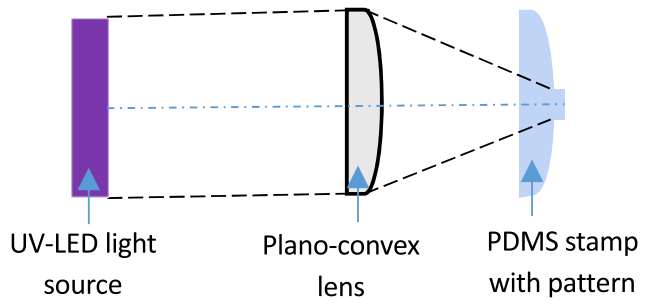

Fig. 1 a Basic model of nanopositioning and nanomeasuring machine with additional rotating nanoimprint tool: 1 metrological frame of NMM-1; 2 rotation stage $\left(\phi_{z}\right) ; 3$ goniometer stage $\left(\phi_{x, y}\right) ; 4$ NIL tool; 5 substrate; 6 mirror corner of NMM-1 (moved in $x, y, z$ ); 7 interferometer of NMM-1; 8 metrological frame of NMM-1. b Detailed schematic of developed NIL tool

of the NMM-1, the Abbe comparator principle is adopted for all axes. This approach avoids first-order positioning errors and linear correction movements for tool rotation and enables orthogonal nanoimprinting on nearly freeformed surfaces.

The 5-degree-of-freedom (DOF) combination of rotational bearings (rotary stage) and segmental rotational bearings (goniometer) provides an optimal solution for miscellaneous challenges. One such challenge is the combination of two rotational axes whose crossing under $90^{\circ}$ is not possible because of working space limitations. An additional crossing angle would lead to the non-independent behavior of the two axes. The combination of a segmentally rotational bearing mounted on a rotational bearing fulfills all requirements without any restrictions in the measurement volume. Therefore, a goniometer stage is mounted on a rotary stage herein [11]. 
The goniometer, which now forms an integral part of the entire rotating mechanism, enables rotation in a precise angular position. Additionally, the goniometer stage provides $\pm 45^{\circ}$ rotation in the $x$-and $y$-directions by using a curved, profiled rail guide and a precision worm gear driven by a servo motor. In combination with the rotary stage, which is active at $360^{\circ}$ in the $z$-axis, the goniometer stage enables ultrahigh resolution for small ranges of motion in micro- and nanopositioning. Thus, the combined motions, i.e., three translational motions of the NPMM and two rotational motions of the goniometer and rotation stage, enable 5-DOF motions.

The optimization of the rotation tool to address a hemisphere orthogonally is realized by determining several possible kinematic variants and then analyzing them by using a parameter-based evaluation system. A high degree of fulfillment is shown by these variants for the rotation of the tool. However, given the unknown structure of the substrate, linear corrections need to be made to address the entire surface of the substrate. If the sample fits the dimensions derived from the given Cartesian volume, then any hemisphere within the characteristic volume can be measured.

The integrated rotating NIL tool is developed on the basis of the basic components of soft UV-NIL (Fig. 1b). It consists of a UV light-emitting diode (LED) light source (Lumitronix LED Technik $\mathrm{GmbH}$ ) with a wavelength of $365 \mathrm{~nm}$ and an intensity of $148 \mathrm{~mW} / \mathrm{cm}^{2}$. A fused silica plano-convex lens is used to focus the light on an area of approximately 20 $\mathrm{mm}^{2}$. The focused light is transmitted through the patterned area of $25 \mathrm{~mm}^{2}$ on the polydimethylsiloxane (PDMS, Sylgard 184 Silicone elastomer) stamp. The stamp has a diameter of $20 \mathrm{~mm}$ and is fixed to the edge of the NIL tool. The developed process is implemented with a resist filling time of $1 \mathrm{~min}$ for the filling of resist into the cavities of the microand nanostructures during molding. Then, resist curing by exposure to UV light for $1 \mathrm{~min}$ is performed. Demolding is subsequently carried out. Imprinting can be implemented multiple times on a single substrate at different angles of rotation. The imprinting process involves the synchronization of the movements of the rotating NIL tool and mirror corner so as to successfully implement the diagonal molding and demolding process (Fig. 2). The control of both tools is realized manually at this stage to ensure coordination.

\subsection{Spray Coating for Plano-Convex Lens Substrate}

Spray coating is performed using EVG 101 spray coater (EV Group). In the case of non-planar surfaces, such as the plano-convex lenses used in this work, a multiple-layer coating is required to achieve good homogeneity and coverage.

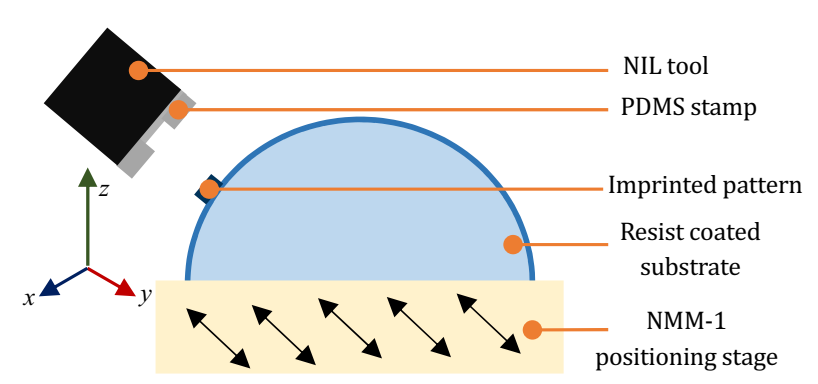

Fig. 2 Orthogonal molding and demolding on edges of curved surface enabled by the diagonal movement (shown by arrows) of the positioning stage

Before starting the spray coating process, the lens is cleaned using acetone and isopropanol and then placed on a hotplate for $10 \mathrm{~min}$ at $115{ }^{\circ} \mathrm{C}$ to remove any moisture from the surface of the lens. It is then coated with an adhesion promoter (TI Prime, Microchemicals $\mathrm{GmbH}$ ) by using spin coating at $4000 \mathrm{r} / \mathrm{min}$ and baked for $2 \mathrm{~min}$ at $115^{\circ} \mathrm{C}$ on the hotplate.

Before starting the process of spray coating using the spray coater, the ultrasonic nozzle to be used is cleaned using acetone. Then, the resist AMONIL MMS4 (AMO $\mathrm{GmbH}$ ) is filled into the syringe pump of the spray coater. After filling the syringe with the resist, the parameters of the spray coater are adjusted. Additional tests on varying geometries show that the individual parameters of the spray coater need to be optimized according to the geometries (length, width, height, and diameter). Two layers of spray coating are completed, and the dispense rate and nozzle flow are changed for each layer. Meanwhile, the high-frequency power and spin speed are kept constant at $0.7 \mathrm{~W}$ and $50 \mathrm{r} /$ min, respectively. For the first layer, a dispense rate of $6 \mu \mathrm{L} / \mathrm{s}$ and nozzle flow rate of $180 \mathrm{dL} / \mathrm{min}$ are set. After the coating of the first layer, a soft bake is performed for $15 \mathrm{~s}$ at $100{ }^{\circ} \mathrm{C}$. The second layer of coating is completed at a dispense rate of $11 \mu \mathrm{L} / \mathrm{s}$ and a nozzle flow rate of $150 \mathrm{dL} / \mathrm{min}$. Thereafter, the lens is again soft baked on the hotplate at $100{ }^{\circ} \mathrm{C}$ for $45 \mathrm{~s}$.

In addition to the aforementioned parameters, the velocity profile of the nozzle is an important parameter influencing the homogeneity of resist coating on non-flat surfaces. The nozzle speed is divided into several indices that define the nozzle speed in different segments of the substrate. During the process, seven out of 15 indices are used on the basis of the geometry of the lens substrate. Pham et al. [12] describe the working mechanism of the spray coater and the effects of the variations of different spray coater parameters. 
Fig. 3 a Soft UV-NIL process at the edge of the plano-convex lens; $\mathbf{b}$ imprinted plano-convex lens at angles of $\mathbf{c} 90^{\circ}$ (top) and $\mathbf{d} 45^{\circ}$ (edge) of the rotating NIL tool

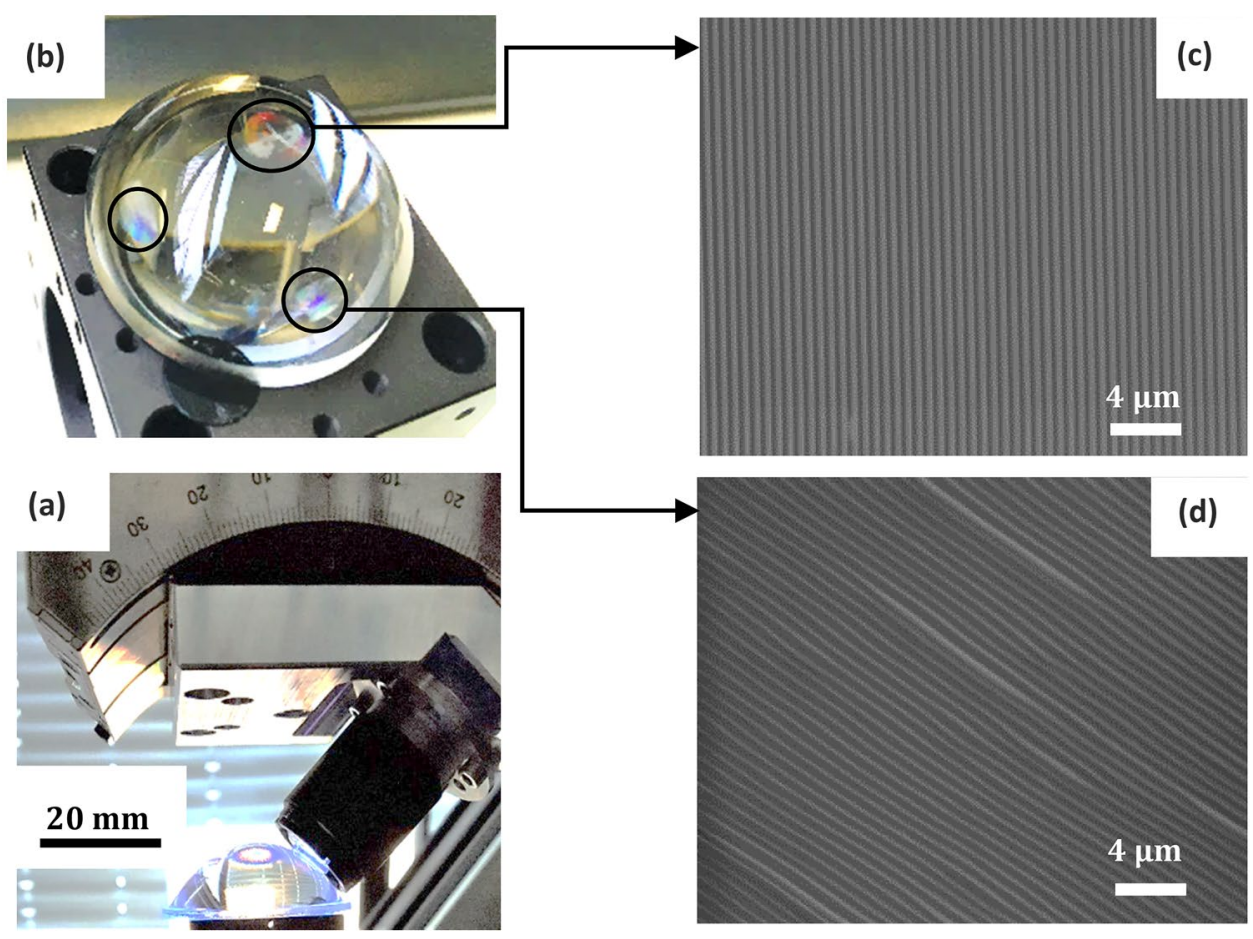

\section{Results and Discussion}

Figure 3 provides an overview of the implemented process. Here, Fig. 3a shows the rotating NIL tool in contact with the substrate during the UV curing stage. The different imprinted areas on the lens surface are shown in Fig. $3 b$. Figure $3 \mathrm{c}, \mathrm{d}$ are the scanning electron microscope images of the imprinted nanostructures on top and at one of the edges of the lens, respectively. The nanostructures have a lateral resolution of $200 \mathrm{~nm}$. The microstructures are imprinted to obtain an imprinted pattern profile and analyze the fidelity of the imprinted patterns on the edges of the lens by using a laser scanning microscope (LSM).
The imprinted microstructures measured at an angle of $45^{\circ}$ on the edge of the lens are found to be uniform and of good fidelity relative to the structure profile of the stamp (Fig. 4a and b). The imprinted structures are further scanned using a profilometer to obtain an enhanced profile of the structures. As a result of the use of different techniques, differences in the units of the scale bar can be observed in the graphs in Fig. 4a, b. The measured differences in the structure heights between the stamp and the imprint at two different steps of the structure profile are measured to be 25 and $33 \mathrm{~nm}$, as derived from Table 1 . These differences are calculated for two different levels of the microstructure, as marked in the graphs in Fig. 4.
Fig. 4 3-D laser scanning microscope images and corresponding structure profiles of (a) imprinted pattern using rotating NIL tool on the edge of the plano-convex lens (measured using a profilometer); $\mathbf{b}$ master pattern on PDMS stamp

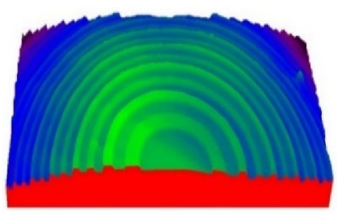

(a)

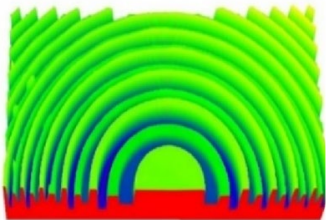

(b)
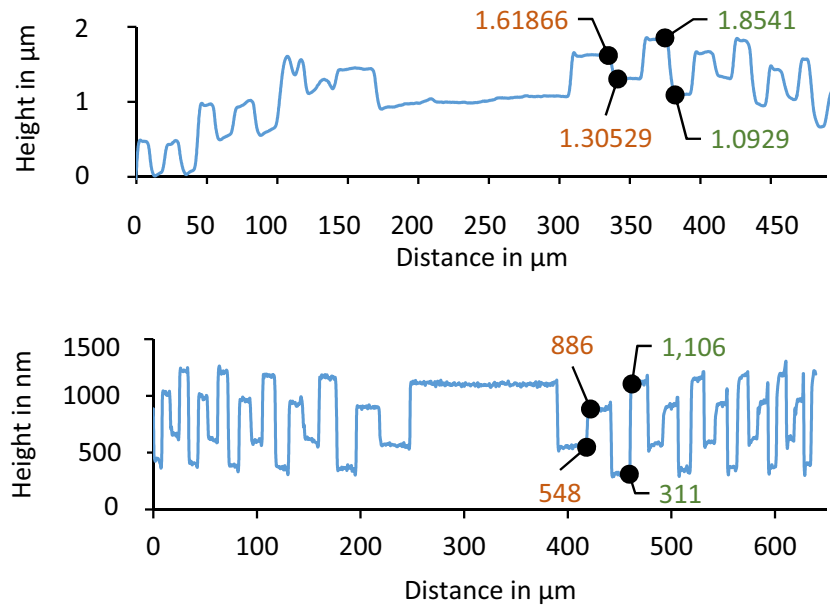
Table 1 Structure height comparison between the stamp and the imprint at two different steps

\begin{tabular}{lcc}
\hline & $\begin{array}{l}\text { Height for Step 1 } \\
(\mathrm{nm})\end{array}$ & $\begin{array}{l}\text { Height } \\
\text { for Step } \\
2(\mathrm{~nm})\end{array}$ \\
\hline Stamp & 338 & 795 \\
Imprint & 313 & 762 \\
Height difference $(\mathrm{nm})$ & 25 & 33 \\
\hline
\end{tabular}

The minimal difference in the structure heights implies the good filling of the resist into the structure cavities, with the pattern almost replicating the stamp. However, as a result of the strong curvature of the macroscopic substrates, their entire surface cannot be patterned uniformly with a single stamping process. Instead, the entire procedure must be divided into individual imprint processes, and the individual surfaces must be stitched together. For nanometer accuracy stitching over the entire surface of the strongly curved substrate, the ultraprecise positioning of the stamp relative to the substrate is necessary. In this case, the highest possible reproducibility of positioning in conjunction with a corresponding in situ measurement of position deviations is indispensable. This factor is realized for the three linear axes of the NMM-1 through the position detection of the mirror corner via laser interferometers and autocollimators. The maximum measured position repeatability of the TCP over the entire rotation range is $\pm 100 \mathrm{~nm}$ for the $x$ - and $y$-directions and $\pm 150 \mathrm{~nm}$ in the $z$-direction [11] [13]. A high positioning accuracy requires the in situ position determination of the rotating tool. Further steps include the final assembly of such a developed system, the integration of the nanoimprinting tool, and the development of the stitching process.

\section{Conclusions}

The newly developed rotating NIL tool, in combination with the NPM machine, allows not only orthogonal imprinting on the edges of highly curved substrates but also imprinting on free-form surfaces with high accuracy and precision. The integration of the two tools results in a good combination of their advantages and provides a unique method for implementing the soft UV-NIL process. On the one hand, the low Young's modulus of the PDMS stamp enables conformal contact for curved surfaces and easy release from imprinted substrates. On the other hand, this factor limits the replication of high-resolution features. With the developed tool, a soft stamp can be used as a hard stamp by placing it orthogonal to the edge of the substrate. Such a setting eliminates the disadvantage of structure bending at high curvatures because a rigid stamp facilitates the replication of high-resolution features [14]. The previously established methods for the NIL process on curved surfaces focus mostly on PDMS stamp formation or the modification of the actual process conditions. However, these processes are focused on small-sized substrates that are mostly in the micrometer or millimeter range. They also neglect the characterization of structures at edges because of the challenges arising from their complexities. With the development of the rotating NIL tool, these facets are explored.

Imprinting is performed on the edges of large planoconvex lens with a diameter of $48.7 \mathrm{~mm}$ and a corresponding height of $18.6 \mathrm{~mm}$ at angles of $45^{\circ}, 60^{\circ}$, and $90^{\circ}$ from the center of rotation of the rotating tool. Additionally, the placement of the substrate on the mirror corner of the NPM machine enables the highly precise diagonal motion of the substrate, which in turn allows orthogonal molding and demolding at different angles. The high resolution and accuracy of the positioning stage ensure a smooth demolding process without shear effects, which is one of the major issues in the NIL process. The imprinted high-resolution nanostructures with good fidelity at the edges of the high curvature substrates depict a desirable outcome given the intricacy of the high-resolution soft UV-NIL process, even on flat substrates.

Thus, based on fundamental concepts of the soft UV-NIL process, together with a high-precision positioning machine, the rotating tool with multiple degrees of freedom combines the best features of each and enables successful orthogonal imprinting process. The further optimization of the process can lead to the enabling of stitching for covering entire surfaces of the substrate.

Acknowledgements The authors gratefully acknowledge the support by the Deutsche Forschungsgemeinschaft (DFG) in the framework of the Research Training Group Tip and Laser-based 3D-Nanofabrication in extended macroscopic working areas (GRK 2182) at the Technische Universität Ilmenau, Germany. The authors would also like to thank Joachim Döll, Rostyslav Mastylo, Johannes Kirchner, Martin Hofmann, David Fischer, and Xinrui Cao for their support.

Funding Open Access funding enabled and organized by Projekt DEAL.

Availability of Data and Material Data available on request from the corresponding author.

Code Availability Not applicable.

\section{Declarations}

Conflict of interest The authors declare that they have no conflicts of interest.

Ethical Approval Not applicable.

Consent to Participate Not applicable. 
Open Access This article is licensed under a Creative Commons Attribution 4.0 International License, which permits use, sharing, adaptation, distribution and reproduction in any medium or format, as long as you give appropriate credit to the original author(s) and the source, provide a link to the Creative Commons licence, and indicate if changes were made. The images or other third party material in this article are included in the article's Creative Commons licence, unless indicated otherwise in a credit line to the material. If material is not included in the article's Creative Commons licence and your intended use is not permitted by statutory regulation or exceeds the permitted use, you will need to obtain permission directly from the copyright holder. To view a copy of this licence, visit http://creativecommons.org/licenses/by/4.0/.

\section{References}

1. Farshchian B, Amirsadeghi A, Hurst SM, Wu J, Lee J, Park S (2011) Soft UV-nanoimprint lithography on non-planar surfaces. Microelectron Eng 88(11):3287-3292. https://doi.org/10.1016/j. mee.2011.07.010

2. Lan H (2018) Large area nanoimprint and applications. Micro/ nanolithography: a heuristic aspect on the enduring technology, $\mathrm{p} 43$

3. Chang JH, Cheng FS, Chao CC, Weng YC, Yang SY, Wang LA (2005) Direct imprinting using soft mold and gas pressure for large area and curved surfaces. J Vac Sci Technol, A: Vac, Surf Films 23(6):1687-1690. https://doi.org/10.1116/1.2073447

4. Chen YP, Lee YP, Chang JH, Wang LA (2008) Fabrication of concave gratings by curved surface UV-nanoimprint lithography. J Vac Sci Technol B: Microelectron Nanometer Struct Process, Measurement, Phenomena 26(5):1690-1695. https://doi.org/10. $1116 / 1.2968702$

5. Cui Z (2008) Nanofabrication. Course notes, ECE, 730.

6. Sreenivasan SV (2017) Nanoimprint lithography steppers for volume fabrication of leading-edge semiconductor integrated circuits.
Microsyst Nanoeng 3:17075. https://doi.org/10.1038/micronano. 2017.75

7. Manske E, Hausotte T, Mastylo R, Machleidt T, Franke KH, Jäger $\mathrm{G}$ (2007) New applications of the nanopositioning and nanomeasuring machine by using advanced tactile and non-tactile probes. Meas Sci Technol 18(2):520. https://doi.org/10.1088/0957-0233/ $18 / 2 / \mathrm{S} 27$

8. Langlotz E, Dontsov D, Schott W (2011) 3D capability for nanopositioning and nanometrology. Laser Photonics Rev 1:36-39

9. Jäger G, Manske E, Hausotte T, Müller A, Balzer F (2016) Nanopositioning and nanomeasuring machine NPMM-200-a new powerful tool for large-range micro-and nanotechnology. Surf Topogr Metrol Prop 4(3):034004. https://doi.org/10.1088/2051672X/4/3/034004

10. Supreeti S, Kirchner J, Hofmann M, Mastylo R, Rangelow IW, Manske E, Hoffmann M, and Sinzinger S (2019) Integrated soft UV-nanoimprint lithography in a nanopositioning and nanomeasuring machine for accurate positioning of stamp to substrate. In Novel Patterning Technologies for Semiconductors, MEMS/ NEMS, and MOEMS 2019 (Vol. 10958, p. 1095819). International Society for Optics and Photonics. https://doi.org/10.1117/ 12.2514832

11. Fern F, Schienbein R, Füßl R, Theska R (2018) Ultra precise motion error measurement of rotation kinematics for the integration in nanomeasuring and nanofabrication machines. In 33rd ASPE Annual Meeting

12. Pham NP, Burghartz JN, Sarro PM (2005) Spray coating of photoresist for pattern transfer on high topography surfaces. J Micromech Microeng. https://doi.org/10.1088/0960-1317/15/4/003

13. Schienbein R, Fern F, Theska R, and Füßl R (2019) On the development and qualification of multiaxial designs of nanofabrication machines with ultra-precision tool rotations. In 2019 euspen's 19th International Conference \& Exhibition, Bilbao, ES

14. Kwon B, and Kim JH (2016) Importance of molds for nanoimprint lithography: hard, soft, and hybrid molds. 2016:6571297. https:// doi.org/10.1155/2016/6571297 